\title{
Characterization of common marmoset (Callithrix jacchus) bone marrow-derived mesenchymal stem cells
}

\author{
Akifumi Kanda, Yusuke Sotomaru, Asako Nobukiyo, Emi Yamaoka, Eiso Hiyama \\ Natural Science Center for Basic Research and Development, Hiroshima University, Hiroshima \\ 734-8551, Japan
}

\begin{abstract}
Mesenchymal stem cells (MSCs) could be useful for regenerative medicine because they can be harvested easily from the bone marrow of living donors and the cells can be differentiated into adipogenic, osteogenic, and chondrogenic lineages in vitro. To apply MSCs for the medical treatment of human diseases as regenerative medicine, detailed experimental characterization of the cells is required. Recently, a New World primate, the common marmoset (Callithrix jacchus), has been widely used as a new human disease model because of its ease of handling and breeding. Although common marmoset MSCs have been established and will be used in preclinical studies of regenerative medicine, the characteristics of these cells remain unclear. Aiming to characterize common marmoset MSCs further, we harvested common marmoset bone marrow-derived cells (cmBMDCs) from the femurs of newborn males. We revealed that the morphology of the cells was similar to common marmoset fibroblasts, and extracellular matrix components, such as gelatin and fibronectin, were effective for their proliferation and formation of colony-forming unit fibroblasts. Furthermore, we were able to differentiate cmBMDCs into adipocytes, osteocytes, and chondrocytes in vitro, and they expressed the MSC markers CD44, CD73, CD90, and CD105, but their expression decreased with increasing passage number. The data demonstrate that cmBMDCs exhibit characteristics of MSCs and thus it would be beneficial to use these cells in preclinical studies. (Folia Histochemica et Cytobiologica 2013, Vol. 51, No. 4, 292-299)
\end{abstract}

Key words: common marmoset; mesenchymal stem cells; extracellular matrix; in vitro differentiation; cell surface proteins

\section{Introduction}

Stem cells are essential to regenerative medicine because of their ability to differentiate into a wide variety of cell types. Mesenchymal stem cells (MSCs), which are derived from bone marrow, are one of the many kinds of stem cells and are known as multipotent stem cells that have the potency to differentiate into mesoderm-derived lineages, such as adipocytes, osteocytes, and chondrocytes [1,2]. MSCs are also easy to handle and are relatively safe for use in rege-

Correspondence address: Y. Sotomaru, Natural Science

Center for Basic Research and Development,

Hiroshima University, 1-2-3 Kasumi, Minami-ku,

Hiroshima 734-8551, Japan;

tel.: +81 82257 5105; fax: +81 822575109 ;

e-mail: yusuke@hiroshima-u.ac.jp nerative medicine because the method used to isolate them from human bone marrow has been established and the cells pose no risk for immune rejection. In addition, the development of tumors in patients who receive implanted MSCs has not been reported yet [3]. Recently, although induced pluripotent stem (iPS) cells have been established from human somatic cells and also do not induce an immune response, these cells have the ability to form tumors in vivo [4]. Due to these advantages of MSCs, they could be used widely in regenerative medicine.

MSCs have been well characterized in humans and mice $[5,6]$. For example, MSCs have a similar morphology to fibroblasts, they have the ability to form colony-forming unit fibroblasts (CFU-F), and they express surface markers such as CD29, CD44, and CD90, but not the hematopoietic and endothelial markers CD34 and CD45 [6, 7]. Despite the stem cell 
characteristics of MSCs, these cells can undergo only a limited number of cell divisions, which is a limitation for their use in regenerative medicine. With an aim to resolve this major problem, immortalized human MSCs have been established; however, these cells gained the ability to form teratomas [8].

The common marmoset (Callithrix jacchus) is a New World non-human primate species and is an important experimental animal to elucidate the mechanisms underlying human diseases and to develop effective therapies [9]. This primate is relatively easy to use in a wide range of research fields in comparison with other experimental models because of its small body size (adult size: 200-500 g), attaining sexual maturity at the age of 1 year, and high breeding efficiency. Actually, a spinal cord injury model has been developed with an aim to find cures for serious diseases [10]. Recently, embryonic stem cells and iPS cells were established from the common marmoset and, in addition, transgenic common marmosets expressing enhanced green fluorescent protein (EGFP) have been generated [11-13]. Thus, these cells and transgenic marmosets will provide important tools for use in preclinical studies.

MSCs have been established from rhesus macaques, cynomolguses, and common marmosets [14-16]. All of these cells have the ability to differentiate into three lineages: adipocytes, osteocytes, and chondrocytes. However, the characteristics of common marmoset MSCs are not well understood. Given this background, we harvested and characterized common marmoset bone marrow-derived cells (cmBMDCs).

\section{Material and methods}

Animals. We used common marmosets obtained from CLEA Japan (EDM: C. Marmoset (Jic); CLEA Japan, Tokyo, Japan) at the Animal Facility of the Natural Science Center for Basic Research and Development, Hiroshima University, and maintained the animals at the Central Institute for Experimental Animals. All experiments were carried out after obtaining permission from the Institutional Animal Care and Use Committee of Animal Experimentation at Hiroshima University.

Harvesting and culture of cmBMDCs and fibroblasts. To harvest cmBMDCs, both epiphyses of the femurs from newborn common marmoset males were cut off and then flushed out using a syringe (23-gauge needle) containing M2 medium (Sigma, St. Louis, MO, USA). The extracted cells were seeded in culture dishes and washed using Dulbecco's modified Eagle's medium (DMEM; Wako, Osaka, Japan) containing $10 \%$ fetal bovine serum (FBS; Invitrogen, Carlsbad, CA, USA) and $100 \mathrm{U} / \mathrm{mL}$ penicillin-streptomycin
(Nacalai Tesque, Kyoto, Japan) to remove floating cells. The adherent cells were expanded in DMEM with $10 \%$ FBS and $100 \mathrm{U} / \mathrm{mL}$ penicillin-streptomycin at $37^{\circ} \mathrm{C}$ in $5 \% \mathrm{CO}$. When the cells were $\sim 70 \%$ confluent, they were dissociated with $0.25 \%$ trypsin (Invitrogen) and passaged in 100-mm dishes.

To harvest common marmoset fibroblasts, the ears of newborn males were minced with scissors and cultured in 35-mm culture dishes containing DMEM with 10\% FBS and $100 \mathrm{U} / \mathrm{mL}$ penicillin-streptomycin at $37^{\circ} \mathrm{C}$ in $5 \% \mathrm{CO}_{2}$. The cells were dissociated with $0.25 \%$ trypsin/EDTA before they reached $100 \%$ confluence.

Proliferation assay. To investigate the effect of extracellular matrix coatings on the proliferation of cmBMDCs, a total of 1,500 cells were seeded in uncoated, $0.1 \%$ gelatin- (Millipore, Temecula, CA, USA), collagen type I-C- (Nitta Gelatin, Tokyo, Japan), and fibronectin-coated (BD Biosciences, Franklin Lakes, NJ) 12-well plates, and trypsinized cells were collected and counted with a counting chamber every 3 days $(n=4)$. This experiment was performed over a period of 8 days. In addition, to examine the effect of the coatings on the proliferation of the cells over a longer period, we measured population doubling levels (PDLs). We seeded 10,000 cells in uncoated, gelatin-, collagen-, and fibronectincoated 12-well plates, and trypsinized cells were collected and counted every 4 days $(n=3)$. PDLs were calculated using the following formula: PDL $=\log _{2}$ (collected cell number / seeded cell number).

In vitro differentiation assay. Adipogenic, osteogenic, and chondrogenic differentiation were induced using the Human Mesenchymal Stem Cell Functional Identification Kit (R\&D Systems, Minneapolis, MN, USA) according to the manufacturer's instructions. Briefly, for adipogenic differentiation, the cells were seeded at a density of $2.1 \times 10^{4} \mathrm{cells} / \mathrm{cm}^{2}$. When the cells were $100 \%$ confluent, they were cultured in adipogenic differentiation medium for 2 weeks. Adipocytes were stained with Oil Red O (Sigma). For osteogenic differentiation, the cells were seeded at a density of $4.2 \times 10^{3} \mathrm{cells} / \mathrm{cm}^{2}$. The next day, when the cells were $\sim 70 \%$ confluent, they were cultured in osteogenic differentiation medium for 2 weeks. Osteocytes were stained with Alizarin Red S (Sigma). For chondrogenic differentiation, $5.0 \times 10^{5}$ cells were seeded in a $15-\mathrm{mL}$ conical tube containing chondrogenic differentiation medium. After 3 weeks, the chondrocytes were fixed with $4 \%$ paraformaldehyde (PFA; Sigma), embedded in paraffin, sectioned, and stained with Toluidine Blue (Sigma).

Colony-forming unit fibroblast assay. To form colonies, 100 cells were seeded in uncoated, gelatin-, collagen-, and fibronectin-coated $100-\mathrm{mm}$ dishes for 2 weeks. After fixing the colonies with $4 \%$ PFA, they were stained with $10 \%$ Giemsa (Merck, Darmstadt, Germany). Colonies that were more than $3 \mathrm{~mm}$ in diameter were counted as propagated cell colonies. 
Immunofluorescence staining. The cells were cultured on 4-well chamber slides coated with $0.1 \%$ gelatin and then fixed with $4 \%$ PFA for $30 \mathrm{~min}$, rinsed in phosphate-buffered saline (PBS), and treated with Protein Block, Serum-Free (DAKO, Carpentaria, CA, USA) for $30 \mathrm{~min}$. Anti-CD34 (AbD Serotec, Kidlington, UK), anti-CD44 (Thermo Fisher Scientific, Fremont, CA, USA), anti-CD73 (Acris Antibodies, Herford, Germany), anti-CD90 (BioLegend, San Diego, CA, USA), and anti-CD105 (GeneTex, Irvine, CA) antibodies were used as primary antibodies. The cells were incubated with the antibodies diluted in Background Reducing Solution (DAKO) for $1 \mathrm{~h}$. After the cells were washed in PBS, they were incubated with Alexa Fluor 568 goat anti-rabbit IgG and Alexa Fluor 488 goat anti-mouse IgG secondary antibodies (Molecular Probes, Eugene, OR, USA) for $1 \mathrm{~h}$. The cells were mounted on glass slides with VECTASHIELD (Vector Laboratories, Burlingame, CA, USA) and observed under a fluorescent microscope (BZ-9000; Keyence, Osaka, Japan). All procedures were performed at room temperature.

Western blotting. To extract proteins from the cells, we used RIPA Buffer (50 mM Tris, pH 7.4, $150 \mathrm{mM} \mathrm{NaCl}$, $1 \%$ Nonidet P-40, $0.1 \%$ SDS, $0.5 \%$ sodium deoxycholate, $1 \mathrm{mM}$ EDTA, pH 8.0) containing protease inhibitor and phosSTOP inhibitor cocktails (Roche Diagnostics GmbH, Mannheim, Germany). The whole cell extracts were centrifuged at $20,879 \times g$ for $20 \mathrm{~min}$ at $4^{\circ} \mathrm{C}$ to gain cell lysates. The lysates $(20 \mu \mathrm{g})$ were separated by 8 or $10 \%$ sodium dodecyl sulfate-polyacrylamide gel electrophoresis and transferred to an Immobilon-P membrane (Millipore). The membrane was blocked with 5\% skim milk (Nacalai) in Tris-buffered saline/Tween-20 (TBST) at room temperature for $1 \mathrm{~h}$ and then incubated with primary antibodies diluted in 5\% skim milk at $4^{\circ} \mathrm{C}$ overnight. The membranes were washed with TBST and then incubated with secondary antibodies at room temperature for $1 \mathrm{~h}$. After washing with TBST, the signals were detected using ECL Western blotting detection reagents or the ECL Plus Western Blotting Detection System (GE Healthcare, Piscataway, NJ, USA). The primary antibodies used were: anti-CD34 (AbD Serotec), anti-CD44 (Thermo), anti-CD73 (Acris Antibodies), anti-CD90 (Biolegend), anti-CD105 (GeneTex), and anti-GAPDH (Sigma). The secondary antibodies used were: anti-rabbit and anti -mouse HRP conjugate (GE Healthcare).

Quantitative real-time PCR. Total RNA was isolated using an RNeasy Mini Kit (QIAGEN, Valencia, CA, USA), and then $1 \mu \mathrm{g}$ of total RNA was reverse transcribed using ReverTra Ace (Toyobo, Osaka, Japan). Quantitative real-time PCR (qPCR) was performed with SYBR premix Ex Taq-II (Takara, Kyoto, Japan) according to the manufacturer's instructions. The expression levels of endogenous mRNA (CD44 and CD90) were measured with the Mx3000P QPCR system (Agilent Technologies,
La Jolla, CA, USA). Primer sequences were as follows: GAPDH, 5'-CATCCCTGAGACACCATGGGGAAG-3' and 5'-GGGATCATTGATGGCGATGACATCC-3'; CD44, 5'-GCAAGGCTTTCAATAGCACCTTGC-3'and 5'-GTTGGGGTGGATCCGAGGAATCAC-3'; and CD90, 5'-CTGGACTGAGCTTCCAGAACCATGA-3' and 5'-GTATTCTCATGGCGGCAGTCCAGAC-3'. The relative expression levels were normalized against GAPDH.

Statistical assessment. The data for CFU-F efficiency were analyzed using the chi-square test with $P<0.05$ considered significant.

\section{Results}

\section{Analysis of the morphology and proliferation of cmBMDCs}

In a previous report, MSCs exhibited a fibroblast-like morphology [14]; therefore, we harvested cmBMDCs from the femur of newborn common marmoset males and performed morphological analysis of the isolated cells. The cells were spindle-shaped and similar to common marmoset fibroblasts (Figure 1A). Next, to investigate the effect of the extracellular matrix components gelatin, collagen, and fibronectin on the proliferation of cmBMDCs, we seeded the cells on matrix-coated plates and counted the cells every 3 days for up to 8 days. However, differences in the effects of these coating on proliferation were not clear in this short period (Figure 1B). In order to clarify their effects, we plotted the PDLs from passage 6 (P6) to $\mathrm{P} 13$. The results show that the proliferation activity of the three extracellular matrices was higher than for uncoated plates; especially, gelatin and collagen were the most effective (Figure 1C).

\section{cmBMDCs differentiate into adipocytes, osteocytes, and chondrocytes in vitro}

MSCs can be differentiated into three lineages: adipocytes, osteocytes, and chondrocytes. To examine the differentiation ability of our established cmBMDCs, the cells were cultured in the relevant differentiation medium. After 2 weeks in adipogenic differentiation medium, small lipid vesicles were visualized and stained with Oil Red $\mathrm{O}$ (Figure $2 \mathrm{~A}$ and $2 \mathrm{~B}$ ). After culturing cmBMDCs in osteogenic differentiation medium for 2 weeks, the cells were positively stained with Alizarin Red S (Figure 2C). After culturing the cells in chondrogenic differentiation medium for 3 weeks, we observed a spherical-shaped pellet and sections of this pellet were stained with Toluidine Blue 


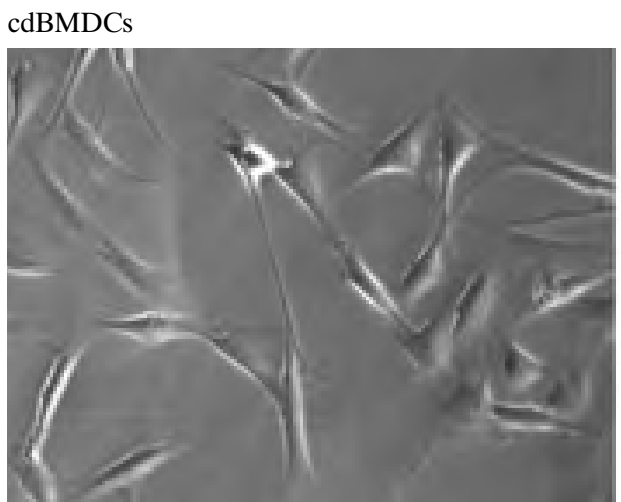

Fibroblasts
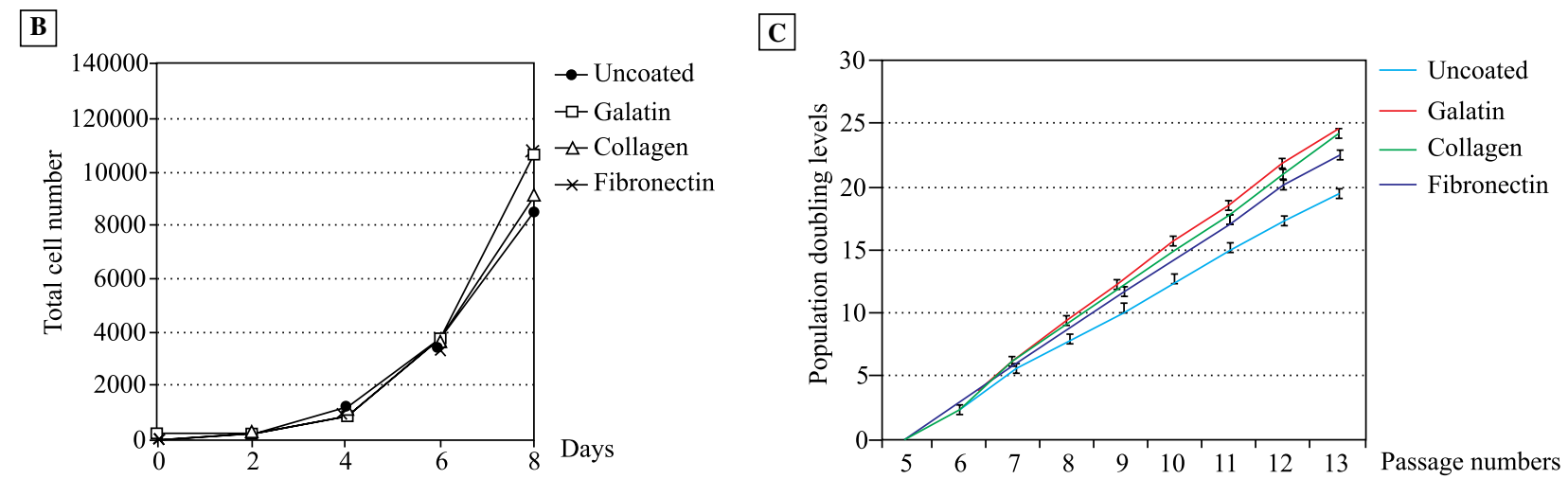

Figure 1. Analysis of morphology and proliferation. A. Comparison of the morphology of common marmoset bone marrow-derived cells (cmBMDCs) and ear-derived fibroblasts. White scale bar: $100 \mu \mathrm{m}$. B. Proliferation assay of cmBMDCs. The cells were seeded on uncoated, gelatin-, collagen-, and fibronectin-coated 12-well plates, and trypsinized cells were collected and counted every 3 days $(n=4)$. C. Determination of the population doubling level. The cells were seeded on uncoated, gelatin-, collagen-, and fibronectin-coated 12-well plates, and trypsinized cells were collected and counted every 4 days $(n=3)$

(Figure 2D and 2E). These results indicate that the cmBMDCs share common characteristics with MSCs such as their ability to differentiate into adipocytes, osteocytes, and chondrocytes in vitro.

\section{Gelatin and fibronectin enhance the ability of cmBMDCs to form CFU-F}

MSCs also have the ability to form CFU-F [15, 17]; therefore, we investigated whether cmBMDCs also have this ability. We found that the cells were able to form cell colonies of various sizes (Figure 3A). To seek the most appropriate extracellular matrix condition to form colonies, we seeded the cells on uncoated, gelatin-, collagen-, and fibronectin-coated dishes, and counted the number of colonies with a diameter of more than $3 \mathrm{~mm}$. The results showed that the number of colonies was significantly increased on gelatin- and fibronectin-coated dishes compared with the uncoated dishes $(P<0.05)$, but not collagen-coated dishes (Figure 3B). Taking these results together with those from the cell proliferation assay (Figure 1B and $1 \mathrm{C}$ ), gelatin and fibronectin effectively induce these abilities in cmBMDCs, but collagen was not suitable for the induction of CFU-F activity.

\section{cmBMDCs express MSC markers}

Human and non-human primate MSCs express cell surface proteins such as CD44, CD73, CD90, and CD105, but not the hematopoietic- and endothelial -specific proteins CD34 and CD45 [7, 15]. To examine whether cmBMDCs express these proteins, we performed immunofluorescence staining using the cells at P10 and P20. We found that cmBMDCs strongly expressed CD44 and CD90, and slightly expressed CD73 and CD105, but faintly expressed CD34, which is negative marker for MSCs (Figure 4A). By contrast, since the fibroblasts expressed CD90 at a very low level, it is speculated that this protein is a positive marker for cmBMDCs. Although we compared the expression of these proteins in cmBMDCs between P10 and P20, there were no apparent differences in their expression. Aiming to clarify the differences, 

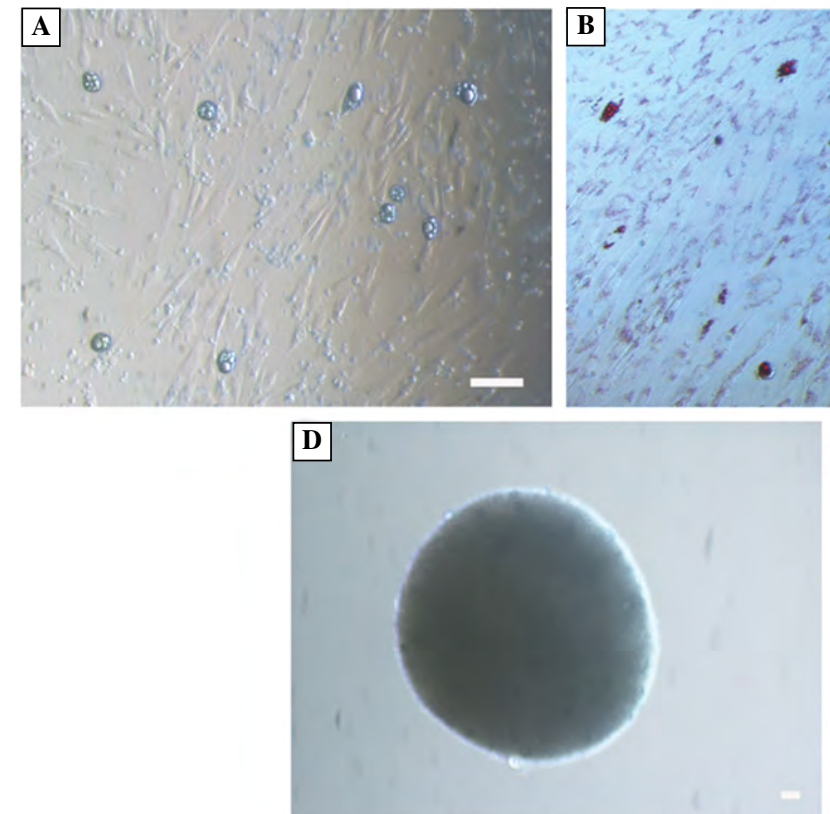
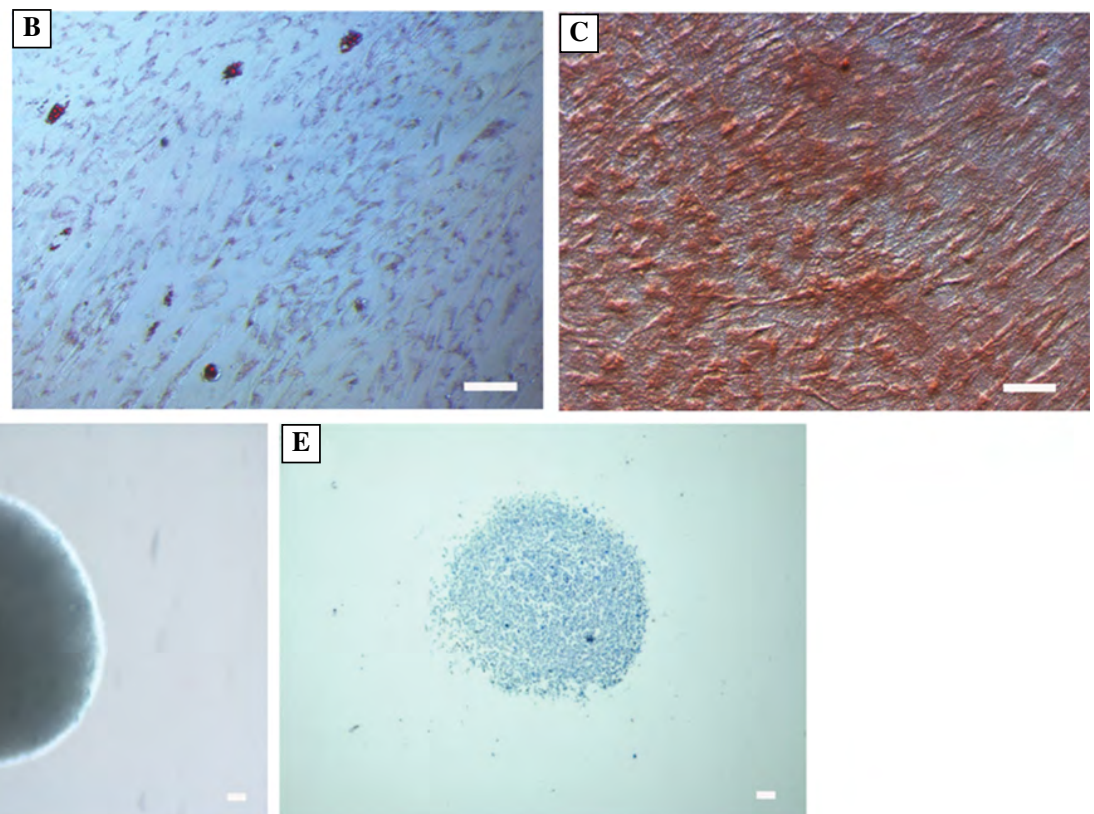

Figure 2. In vitro differentiation of cmBMDCs. Adipogenic differentiation was induced using adipogenic differentiation medium. A. Morphology of adipocytes. B. Adipocytes were stained with Oil Red O. C. Osteocytes induced using osteogenic differentiation medium were stained with Alizarin Red S. Chondrogenic differentiation was induced using chondrogenic differentiation medium. D. Morphology of chondrocytes. E. Chondrocytes were stained with Toluidine Blue. White scale bar: $100 \mu \mathrm{m}$

$\mathbf{A}$

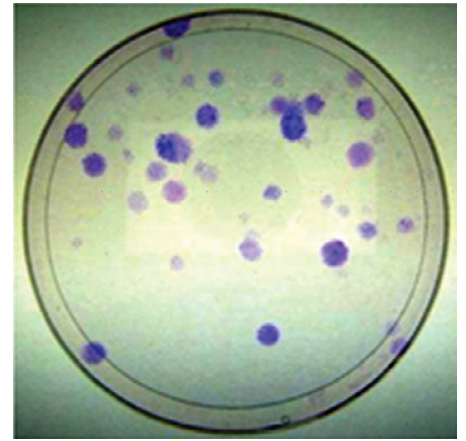

$\mathbf{C}$

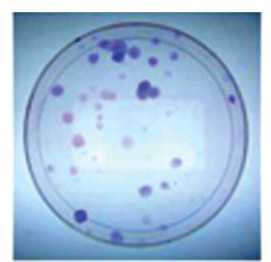

Uncoated

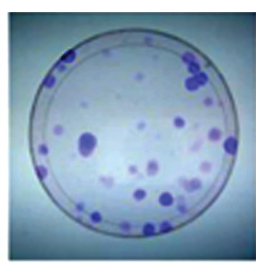

Gelatin
B
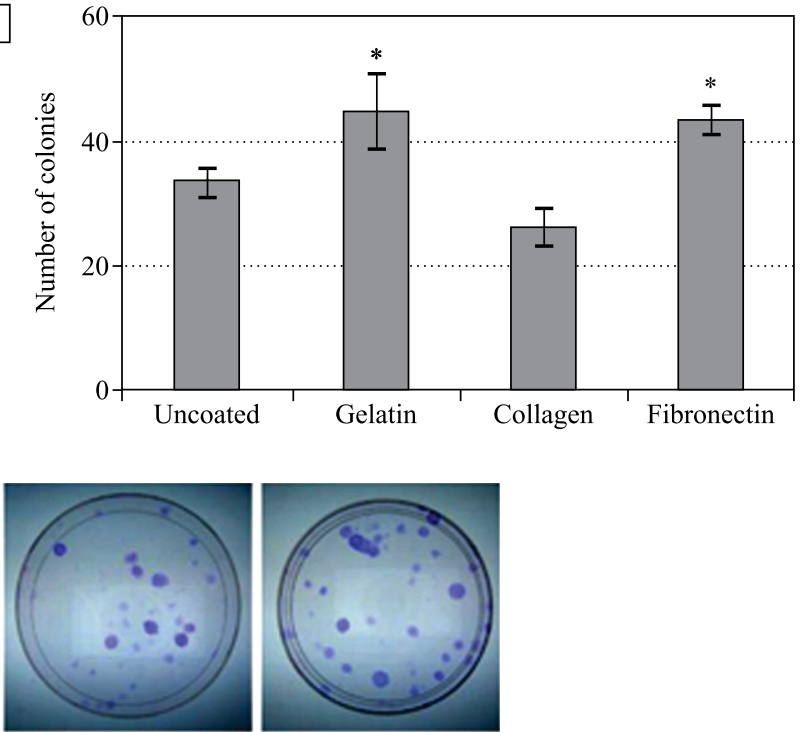

Collagen

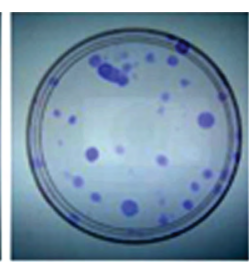

Fibronectin

Figure 3. Colony-forming unit fibroblast assay of cmBMDCs. A. Giemsa-stained colonies cultured on an uncoated dish. B. The graph shows the quantification of the number of stained colonies $\geq 3 \mathrm{~mm}$ in diameter. The number of colonies was significantly increased by gelatin and fibronectin $\left({ }^{*} P<0.05\right)$, but not by collagen, compared to cells cultured on uncoated dishes $(n=4)$. The data are represented as mean $\pm \operatorname{SEM}(n=4) .{ }^{*} P<0.05$. C. The figure shows Giemsa-stained colonies cultured on uncoated, gelatin-, collagen-, and fibronectin-coated dishes

the expression levels of these proteins were evaluated P20. Conversely, the expression of these proteins was using Western blotting. In cmBMDCs, the expression lower in fibroblasts at P10 than in cmBMDCs at P10 of CD44, CD73, CD90, and CD105 was decreased by (Figure 4B). This suggests that the expression of these 
$\mathbf{A}$

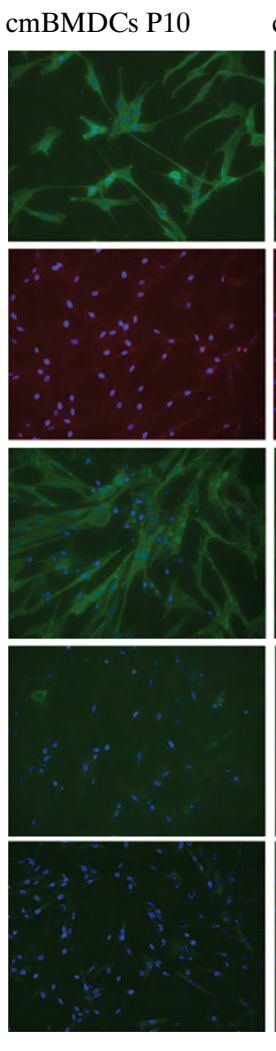

B
cmBMDCs P20
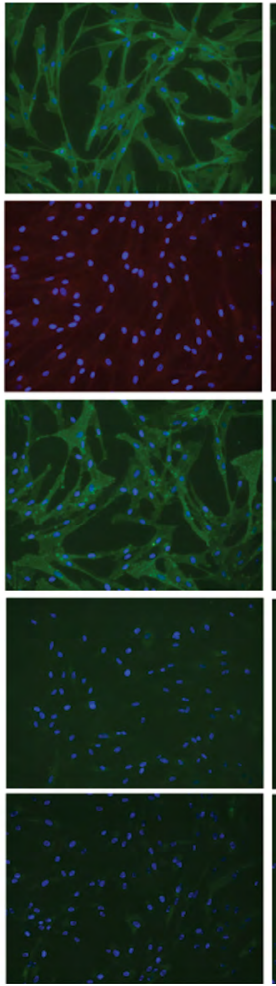

Fibroblasts P12
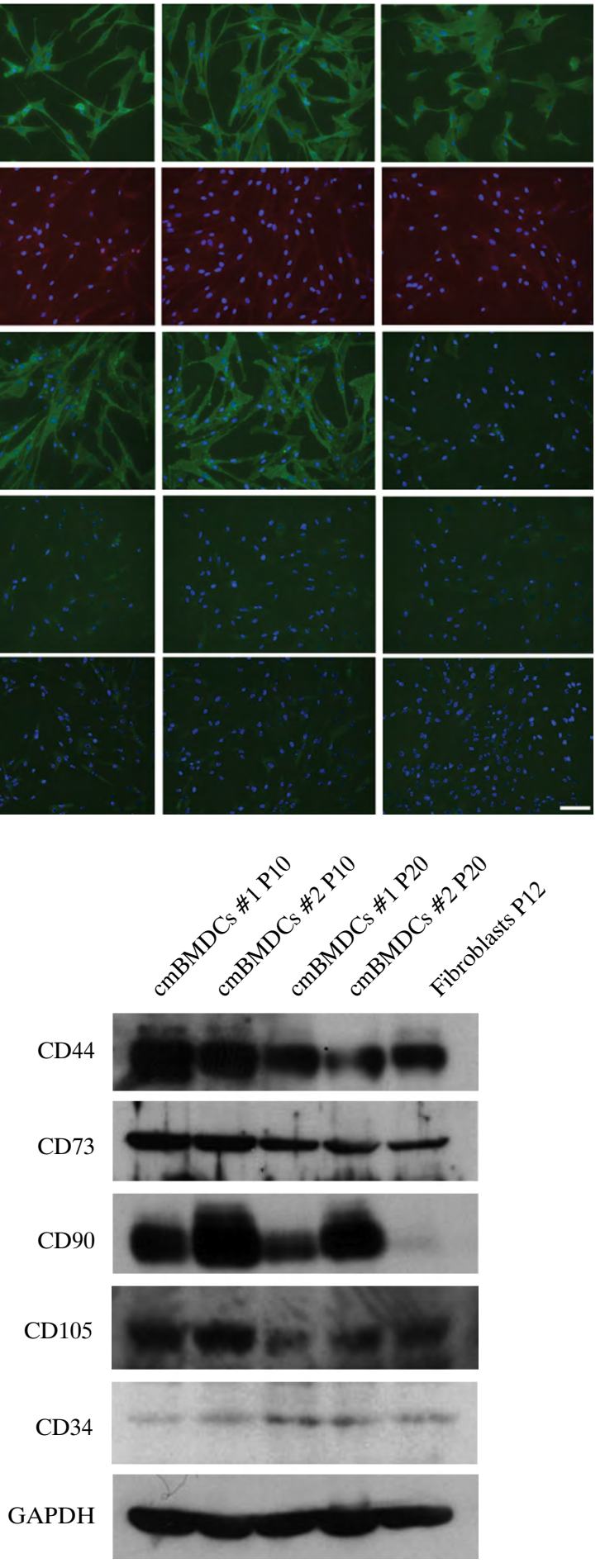

cell surface proteins tends to decrease with increasing passage number. However, on the contrary, CD34 was barely expressed in cmBMDCs at P10 and was slightly increased in cmBMDCs at P20 (Figure 4B).

To verify the results of the Western blotting analysis, we compared the expression levels of CD44 and CD90 mRNA at P10 and P20 by qPCR analysis. As
C
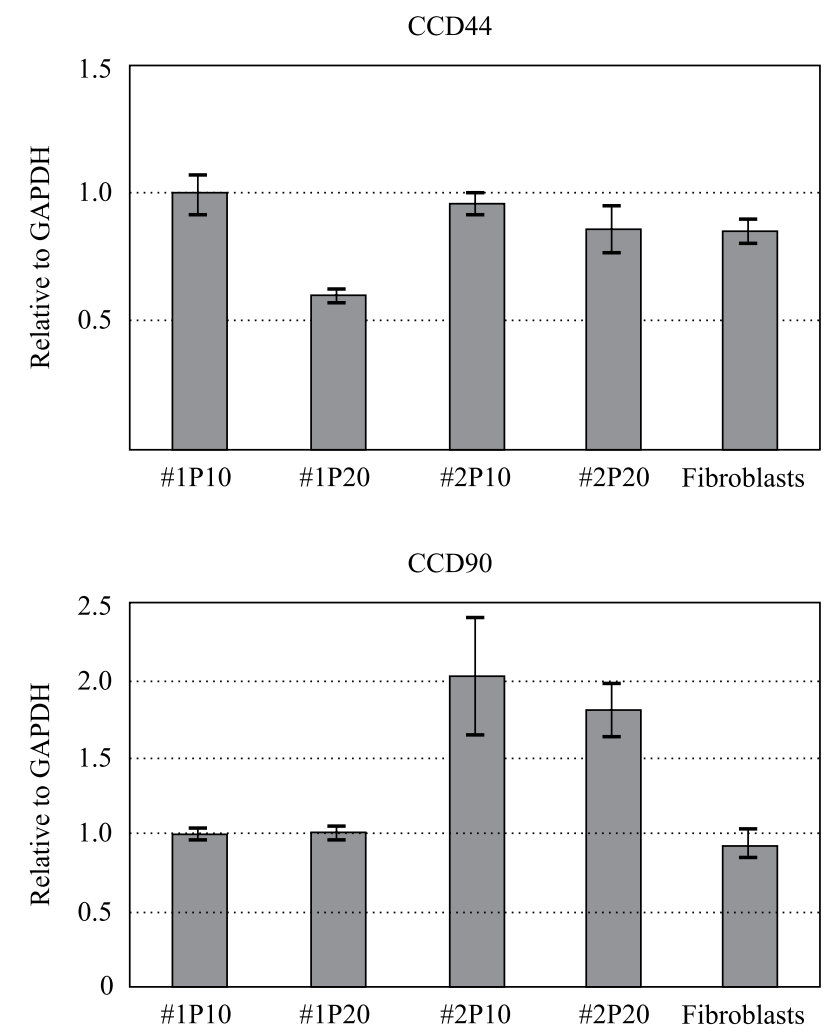

Figure 4. Expression of cell surface markers in cmBMDCs and fibroblasts. A. Immunofluorescence staining of cmBMDCs and fibroblasts. The cells were stained to visualize CD44, CD90, CD105, CD34 (green), and CD73 (red). The nuclei were counterstained with DAPI (blue). White scale bar: $100 \mu \mathrm{m}$. B. Western blotting analysis of CD34, CD44, CD73, CD90, CD105, and GAPDH (B). C. qPCR analysis of CD44 and CD90 mRNA. Levels of mRNA are normalized to GAPDH

a result, there was no significant change in the expression of each mRNA with increasing passage number (Figure 4C), suggesting the data are not consist with those from Western blotting analysis.

\section{Discussion}

MSCs are very attractive multipotent stem cells because of their ability for self-renewal and multi-lineage differentiation, and they are also easy to harvest from bone marrow. Recently, common marmosets have been noted as a new non-human primate model of human diseases, and have been used to assess preclinical cell therapy and stem cell therapy, including gene targeting, to elucidate how to treat intractable diseases [18-21]. Common marmoset MSCs would also be useful stem cells in preclinical studies; therefore, the characterization of these cells is essential to develop the research field of regenerative medicine. 
In this study, we harvested cmBMDCs from the femur of neonatal common marmosets and characterized the isolated cells. PDL analysis revealed that extracellular matrix components such as gelatin and collagen stimulate the proliferation of cmBMDCs. A previous report indicated that collagen I promoted the proliferation of human MSCs in an osteogenic differentiation environment due to the activation of the Akt and ERK signaling pathways, which increase cell proliferation; thus, gelatin and collagen may stimulate the proliferation of cmBMDCs [22]. In contrast to the PDL analysis, collagen did not increase the formation of CFU-F colonies, which indicates that collagen is dispensable for increasing this activity in these cells.

Additional analysis of the characteristics of $\mathrm{cmB}$ MDCs revealed that the cells were able to differentiate into adipocytes, osteocytes, and chondrocytes in vitro and that they expressed the primate MSC markers CD44, CD73, CD90, and CD105, while the protein expression of these markers decreased with increasing passage number. As a possible cause of this decreased expression, contaminating fibroblasts are found in bone marrow and express low levels of MSC markers; another possibility is that the expression level of MSC markers decreases during the long-term culture of human MSCs [23-25]. This decrease in expression indicates that long-term culturing induces changes in the expression of these markers; therefore, cmBMDCs may undergo a similar change in their expression pattern. We investigated whether this decrease could be confirmed at the mRNA level using CD44 and CD90, but interestingly their expression was decreased less than their protein expression. This difference in expression may be the cause of the stability, half-life, and translational efficiency of surface proteins. We also attempted to detect CD45, but were unable to detect a signal (data not shown) because the antibodies were considered to be unable to recognize common marmoset CD45 or that cmBMDCs and fibroblasts do not express this protein. To clarify this problem, we should try to use other CD45 antibodies that can recognize it.

It is important to know the characteristics of cmBMDCs to develop preclinical studies using the common marmoset. In the present study, the characteristics of the cmBMDCs were close to those isolated from humans and other primates in terms of their in vitro differentiation potency and expression of cell surface markers; the only exception was their different pattern of CD34 expression. Therefore, cmBMDCs would provide helpful information in preclinical studies. In general, MSCs are harvested from living individuals, but we failed to isolate these cells from living common marmosets because of their small body size. Previously, a perfusion method to collect MSCs from the ilium of living cynomolgus monkeys using PBS was reported [26]. In the future, we plan to investigate whether this method can also be used to collect cmBMDCs. As an additional improvement after collecting cmBMDCs, eliminating contaminating fibroblasts from the bone marrow is important. In humans, to avoid this contamination, a cell sorting system using a combination of human MSC- and fibroblast-positive antibodies was developed [27]. However, given the similarities in the properties of MSCs and fibroblasts, complete separation of these cells is difficult; hence, it is important to identify cmBMDC- or fibroblast-positive antibodies to distinguish between these cells. Our data suggest that CD90 has the potential to be a candidate protein to distinguish between these cells because its expression was markedly different in cmBMDCs and fibroblasts. We also intend to investigate whether cmBMDCs maintain MSC characteristics beyond P20, such as self-renewal, proliferation activity, and cell surface protein expression.

\section{Acknowledgements}

We thank S. Shiozawa (Department of Physiology, Keio University School of Medicine) for technical advice and S. Sakatani for technical assistance. We also thank T. Hatakeyama, H. Nishimoto, and T. Nojiri for providing animal care. A part of this study was supported by 'Highly creative animal model development for brain sciences' carried out under the Strategic Research Program for Brain Sciences by the Ministry of Education, Culture, Sports, Science and Technology of Japan.

\section{References}

1. Pittenger MF, Mackay AM, Beck SC et al. Multilineage potential of adult human mesenchymal stem cells. Science. 1999;284:143-147.

2. Chen $\mathrm{X}, \mathrm{Xu} \mathrm{H}, \mathrm{Wan} \mathrm{C}$ et al. Bioreactor expansion of human adult bone marrow-derived mesenchymal stem cells. Stem Cells. 2006;24:2052-2059.

3. Weissman IL. Translating stem and progenitor cell biology to the clinic: barriers and opportunities. Science. 2000;287: 1442-1446.

4. Takahashi K, Tanabe K, Ohnuki M et al. Induced of pluripotent stem cells from adult human fibroblasts by defined factors. Cell. 2007;131:861-872.

5. Chamberlain G, Fox J, Ashton B et al. Concise review: mesenchymal stem cells: their phenotype, differentiation capacity, immunological features, and potential for homing. Stem Cells. 2007;25:2739-2749.

6. Soleimani M, Nadri S. A protocol for isolation and culture of mesenchymal stem cells from mouse bone marrow. Nat Protoc. 2009;4:102-106.

7. Liu F, Akiyama Y, Tai S et al. Changes in the expression of CD106, osteogenic genes, and transcription factors involved 
in the osteogenic differentiation of human bone marrow mesenchymal stem cells. J Bone Miner Metab. 2008;26:312-320.

8. Yamaoka E, Hiyama E, Sotomaru Y et al. Neoplastic transformation by TERT in FGF-2-expanded human mesenchymal stem cells. Int. J Oncol. 2011;39:5-11.

9. Mansfield K. Marmoset models commonly used in biomedical research. Comp Med. 2003;53:383-392.

10. Kitamura K, Fujiyoshi K, Yamane J et al. Human hepatocyte growth factor promotes functional recovery in primates after spinal cord injury. PloS One. 2011;6:e27706.

11. Wu Y, Zhang Y, Mishra A et al. Generation of induced pluripotent stem cells from newborn marmoset skin fibroblasts. Stem Cell Res. 2010;4:180-188.

12. Tomioka I, Maeda T, Shimada $\mathrm{H}$ et al. Generating induced pluripotent stem cells from common marmoset (Callithrix jacchus) fetal liver cells using defined factors, including $\operatorname{Lin} 28$. Genes Cells. 2009;15:959-969.

13. Sasaki E, Suemizu H, Shimada A et al. Generation of transgenic non-human primates with germline transmission. Nature. 2009;459:523-527.

14. Izadpanah $\mathrm{R}$, Joswig $\mathrm{T}$, Tsien $\mathrm{F}$ et al. Characterization of multipotent mesenchymal stem cells from the bone marrow of rhesus macaques. Stem Cells Dev. 2005;14:440-451.

15. Ke H, Wang $\mathrm{P}, \mathrm{Yu}$ W et al. Derivation, characterization and gene modification of cynomolgus monkey mesenchymal stem cells. Differentiation. 2009;77:256-262.

16. Bernemann I, Mueller T, Blasczyk R et al. Colonization of collagen scaffolds by adipocytes derived from mesenchymal stem cells of the common marmoset monkey. Biochem Biophys Res Commun. 2011;411:317-322.

17. Chase LG, Lakshmipathy U, Solchaga LA et al. A novel serum-free medium for the expansion of human mesenchymal stem cells. Stem Cell Res Ther. 2010;1:8.

18. Hibino H, Tani K, Ikebuchi $\mathrm{K}$ et al. The common marmoset as a target preclinical primate model for cytokine and gene therapy studies. Blood. 1999;93:2839-2848.
19. Iwanami A, Kaneko S, Nakamura M et al. Transplantation of human neural stem cells for spinal cord injury in primates. J Neurosci Res. 2005;80:182-190.

20. Fujiyoshi K, Yamada M, Nakamura $\mathrm{M}$ et al. In vivo tracing of neural tracts in the intact and injured spinal cord of marmosets by diffusion tensor tractography. J Neurosci. 2007;27:11991-11998.

21. Shiozawa S, Kawai K, Okada $\mathrm{Y}$ et al. Gene targeting and subsequent site-specific transgenesis at the $\beta$-actin (ACTB) locus in common marmoset embryonic stem cells. Stem Cells Dev. 2011;20:1587-1599.

22. Tsai KS, Kao SY, Wang CY et al. Type I collagen promotes proliferation and osteogenesis of human mesenchymal stem cells via activation of ERK and Akt pathways.J Biomed Mater Res A. 2010;94:673-682.

23. Wagner W, Horn P, Castoldi M et al. Replicative senescence of mesenchymal stem cells: a continuous and organized process. Plos One. 2008;21:e2213.

24. Jung EM, Kwon O, Kwon KS et al. Evidences for correlation between the reduced VCAM-1 expression and hyaluronan synthesis during cellular senescence of human mesenchymal stem cells. Biochem Biophys Res Commun. 2011;404:463-469.

25. Bagriacik EU, Yaman M, Haznedar R et al. TSH-induced gene expression involves regulation of self-renewal and differentiation-related genes in human bone marrow-derived mesenchymal stem cells. $J$ Endocrinol. 2012;212:169-178.

26. Kushida $\mathrm{T}$, Inaba M, Ikebukuro $\mathrm{K}$ et al. Comparison of bone marrow cells harvested from various bones of cynomolgus monkeys at various ages by perfusion or aspiration methods: a preclinical study for human BMT. Stem Cells. 2002;20:155-162.

27. Halfon S, Abramov N, Grinblat B et al. Markers distinguishing mesenchymal stem cells from fibroblasts are downregulated with passaging. Stem cells Dev. 2011;20:53-66.

Submitted: 3 April, 2013

Accepted after reviews: 21 November, 2013 